
allemande

48-2 | 2016

Les espaces publics des pays germanophones, des espaces publics transnationaux?

Transnationale Öffentlichkeit zwischen Mobilisierung und Rückzug am Beispiel von Hakim Bey (1945-) und Gustav Landauer (1870-1919)

\title{
Catherine Repussard
}

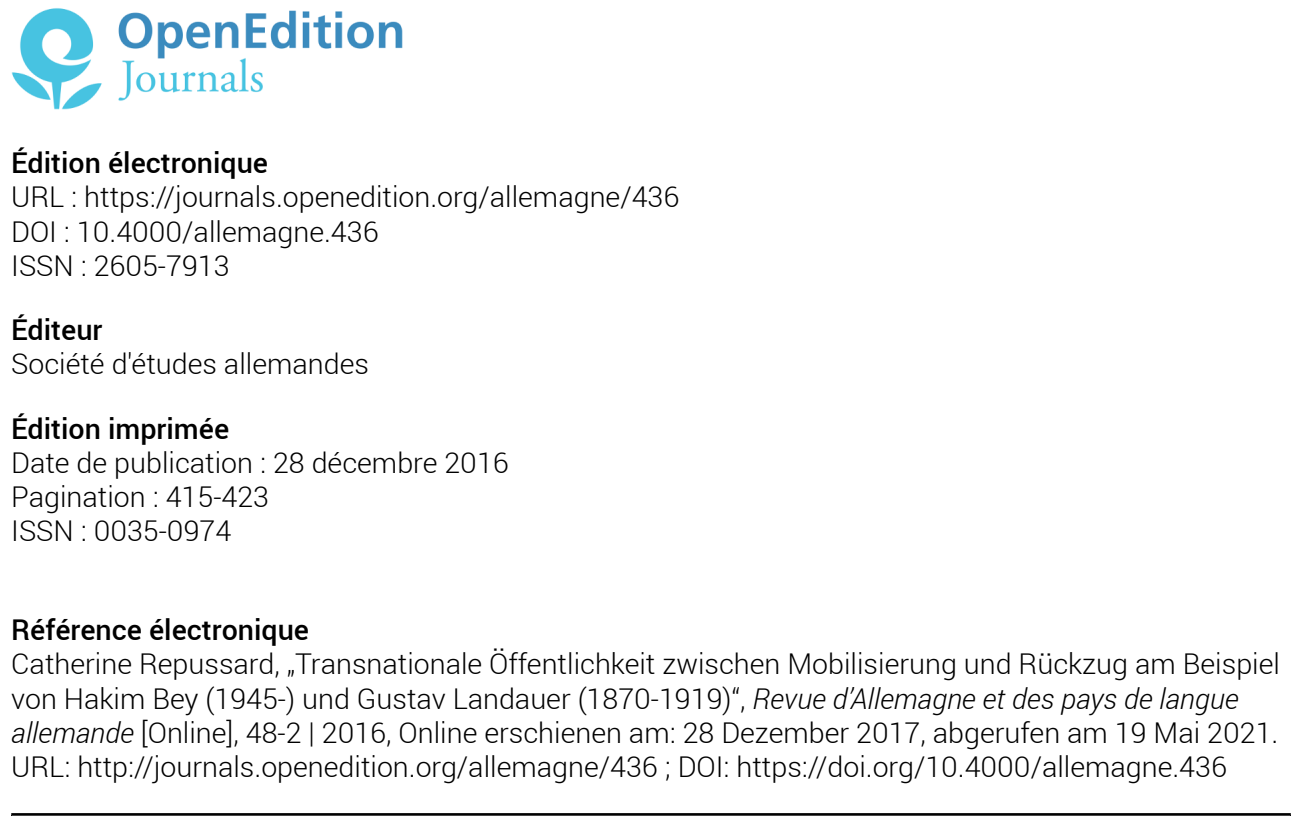

Revue d'Allemagne et des pays de langue allemande 


\title{
Transnationale Öffentlichkeit zwischen Mobilisierung und Rückzug am Beispiel von Hakim Bey (1945-) und Gustav Landauer (1870-1919)
}

- Catherine Repussard*

\author{
„Auch die Vergangenheit ist Zukunft.“ \\ Gustav Landauer, Essays zum Anarchismus \\ „Gustav Landauer ist ein Heiliger." \\ Hakim Bey, TAZ
}

Wird das Thema „transnationale Öffentlichkeit“ bzw. „germanophone transnationale Öffentlichkeit" behandelt, stellt sich von vorn herein die Frage, ob es eigentlich noch „Sinn macht“ in unserer globalisierten Technomedienwelt, die de facto transnational ist, von einer, germanophonen` Öffentlichkeit zu sprechen. Wird beispielsweise zum Thema „transnationale Öffentlichkeit“ recherchiert, fällt einem sofort auf, wie eng der Begriff mit dem Aktivismus der Antiglobalisierungsbewegung verbunden ist, die sich grundsätzlich als transnationale Protestbewegung versteht. Öffentlichkeit entspricht in unserer Analyse der öffentlichen Meinungs- und Willensbildung, im Sinne von Axel Montenbruck, der unter dem Begriff Öffentlichkeit ein kollektives Interesse versteht, mit anderen Worten ein res-publica. Begriffe wie „die Allgemeinheit, die Kollektivität, die Sozialität und die Versammlung zielen auf eine Bündelung von Einzelinteressen und Einzelwesen zu etwas „Gemeinsamem“ ab. Sie alle beschreiben Aspekte von menschlichen Gemeinschaften. Die Öffentlichkeit ist ferner zumeist mit Orten, wie dem Forum, dem Gericht und der Versammlung im Freien und ohne Waffen, verbunden. Die Öffentlichkeit lässt auf diese Weise die Allgemeinheit in einer konkreten Form sichtbar werden und verschafft ihr einen eigenen Raum “(1). Eigentlich kommt deutlich zum Vorschein, wie sehr transnationale Öffentlichkeit auch mit dem Versuch zu tun hat, die aus der Zivilgesellschaft stammenden politischen Alternativen

* Maître de conférences HDR à l'Université de Strasbourg, EA 1341 Études germaniques.

1 Axel Montenbruck, Zivilisation. Eine Rechtsanthropologie. Staat und Mensch, Gewalt und Recht, Kultur und Natur, Berlin, Universitätsbibliothek der Freien Universität Berlin, 2010, S. 291. 
zu fördern und somit die Protestbewegungen aller Länder wieder zusammenzuknüpfen, in einem Zeitalter, in dem sich das Empire im Sinne von neuer Weltordnung, um den Titel des „Epochenbuchs“ - wie es Slavoj Žižek in der New York Times schrieb (2003) - von Michael Hardt und Antonio Negri ${ }^{(2)}$ wiederaufzunehmen, sich vor unseren Augen ausdehnt, wie in ihrem Werk behauptet wird, immer weiter. Das Empire als transnationale technokapitalistische Macht - soll dann auch zur ,Gleichschaltung aller Länder führen, zu einer Art globaler Uniformität auf mondialer Ebene jenseits jeder staatlichen Souveränität ${ }^{(3)}$, oder anders formuliert zu einer „Interkulturation“ der Welt ${ }^{(4)}$. Transnationale Öffentlichkeit kann also sowohl die ,Totalität', bezeichnen, die den ständigen Zugriff der kapitalistischen Medien- und Warengesellschaft auf alle Aspekte des menschlichen Lebens betrifft, wie auch die transnationale Resistenz der Multitudes $^{(5)}$ (sprich der Weltmassen). Den Begriff, Resistenz', der vom Hitlerspezialisten Ian Kershaw ${ }^{(6)}$ geprägt wurde, um die Unmöglichkeit einer frontalen Opposition in der Hitlerzeit zu thematisieren, möchte ich auch wieder aufnehmen, da ein ähnliches Verhalten auch für die weltweiten Alternativbewegungen unserer Zeit charakteristisch ist, die von einer frontalen Konfrontation mit dem bereits erwähnten Empire nichts zu erwarten haben. Zusammenfassend hat transnationale Öffentlichkeit mit transnationaler Resistenz zu tun, mit einer Resistenz also auch in Form einer aus der Zivilgesellschaft entstandenen gegenkulturellen Bewegung. Letztere ist für meine Begriffe keineswegs eine Neuerscheinung. Diese Bemerkung bezieht sich auf diesen Beitrag, der schließlich beabsichtigt, dem Konzept „transnationale Öffentlichkeit“ auch eine diachronische oder genealogische Dimension zu verleihen.

Neuigkeiten sind nämlich selten. Die gerade erwähnten zeitgenössischen Protestbewegungen rufen andere Zeiten in Erinnerung, genauer die Epoche um 1900, als im deutschsprachigen Raum - aber nicht nur ${ }^{(7)}$ - sich Reformbewegungen ${ }^{(8)}$ durchsetzten, die auch als transnationaler Protest im Rahmen einer transnationalen Öffentlichkeit gegen das kommende liberale Technomedienzeitalter - wie wir es heute formulieren würden, damals wurde einfacher von „Fortschritt“ gesprochen - zu verstehen ist. Es geht mir deshalb darum, eine diachronische Dimension der transnationalen Öffentlichkeit zu thematisieren anhand des Engagements zweier Figuren der Alternativität, deren epochale ,Resonanz' deutlich zum Ausdruck kommt: Gustav

2 Michael Hardt, Antonio Negri, Empire: Die neue Weltordnung, Frankfurt am Main, Campus, 2003.

3 Kurz Robert, Weltordnungskrieg, Das Ende der Souveränität und die Wandlungen des Imperialismus im Zeitalter der Globalisierung, Bad Honnef, Horlemann Verlag, 2003.

4 Jacques Demorgon, L'interculturation du monde, Paris, Anthropos, 2000.

5 Michael Hardt, Antonio Negri, Multitude: Krieg und Demokratie im Empire, Frankfurt am Main, Campus, 2004.

6 Ian Kershaw, Hitler 1933-1945, München, Pantheon Verlag, 2009.

7 Vgl. Thomas Rohkrämer, „Gab es eine Lebensreform in England?“, in: Marc Cluet, Catherine Repussard, Lebensreform ou la dynamique sociale de l'impuissance politique / Die Lebensreform oder die soziale Dynamik der politischen Ohnmacht, Tübingen, Francke, 2013, S. 319-334.

8 Vgl. Diethart Kerbs, Jürgen Reulecke et al. (Hg.), Handbuch der Reformbewegungen 1880-1933, Wuppertal, Peter Hammerlag, 1998; Kai Buchнolz, Rita Latocha, Hilke Peckmann, Klaus WolBert (Hg.), Die Lebensreform. Entwürfe zur Neugestaltung von Leben und Kunst um 1900, Bd. 1, Darmstadt, Häuser, 2001; Cluet/Repussard, La Lebensreform ou la dynamique sociale de l'impuissance politique (Anm. 7). 
Landauer ${ }^{(9)}$, der für den ,romantischen Anarchisten' schlechthin gilt und Hakim Bey, eigentlich Peter Lamborn Wilson ${ }^{(10)}, 1945$ in New York geboren, der zur transnationalen Protestfigur geworden ist. Beide Figuren ziehen eine ähnliche Form der Resistenz vor: Absonderung durch Gemeinschaft ${ }^{(11)}$ für Landauer und den Rückzug in TAZ ${ }^{(12)}$ (temporäre autonome Zonen) für Hakim Bey, in der die gesellschaftlichen Regeln und Machtverhältnisse außer Kraft gesetzt sind oder absichtlich missachtet werden. Für beide liegt der Kern des Protests im Versuch des Aussteigens und in der Entwicklung von abgesonderten Zonen, die dem Einzelnen die Möglichkeit bieten, sich frei oder zumindest freier zu entfalten. Um 1900 wie auch um 2000 geht es schließlich den Alternativen immer noch darum: gemeinsam das Leben umweltfreundlich aber auch ethisch-religiös neu zu gestalten.

Der alternative Druck, der um 1900 im Kontext der Reform- bzw. Lebensreformbewegungen zutage trat und die bestehende Gesellschaftsordnung in Frage stellte, ist gekennzeichnet durch die Suche nach einem vollständigen Ausgleich zwischen Mensch und Umwelt bzw. Weltall. Diese Bewegung behauptet sich als Reform des Einzelnen (Selbstreform) innerhalb eines neu ersonnenen Gemeinwesens. Sie fokussiert auf die Notwendigkeit, die harmonische und naturgemäße Entwicklung des Einzelnen zu ermöglichen, im Rahmen eines gemeinschaftlichen Ganzen, wodurch die komplexe Frage der engen Beziehungen zwischen Einzelwesen und Ganzheit aufgeworfen wird.

9 Gustav Landauer (1870-1919). 1892 gründete er mit Bruno Wille und Fritz Mauthner die Neue freie Volksbühne, die das proletarische Massenpublikum mit Appellen an das neuentdeckte Seelenleben von innen her umwandeln wollte. Er war auch als Journalist für die Zeitschriften Die Zukunft, Die Gesellschaft und Das Neue Jahrhundert tätig. 1908 gründete er zusammen mit Martin Buber und Erich Mühsam den Sozialistischen Bund. Im November 1918 stellte sich Landauer als Beauftragter für Volksaufklärung der Regierung Eisner in den Dienst der Münchner Revolution. Beim Einmarsch der gegenrevolutionären Truppen wurde er verhaftet und im Gefängnis ermordet. Zu Landauer: Ulrich LINSE, Gustav Landauer und die Revolutionszeit 1918-1919, Berlin, Kramer, 1974; Martin BuBER, Pfade in Utopia. Über Gemeinschaft und deren Verwirklichung. Eine intensive Auseinandersetzung mit Landauers Ideen, 1950; Wolf SIEGBERT (Hg.), Auch die Vergangenheit ist Zukunft, Frankfurt am Main, Luchhterhand, 1989. Auf französisch: Anatole LUCET, „Gustav Landauer: le devenir révolutionnaire comme alternative anarchiste“, in: Catherine Repussard (Hg.), De la Lebensreform à l'Altermondialisme. Métamorphoses de l'alternativité? Von der Lebensreform zur Antiglobalisierungsbewegung. Metamorphose der Alternative? Recherches Germaniques, Hors-série n 11, Strasbourg, 2016.

10 Peter Lamborn Wilson (besser bekannt unter dem Pseudonym) oder Hakim Bey sieht sich selbst in der Tradition islamischer Mystiker und Ketzer (Sufis, Assassinen) und sympathisiert mit historischen libertären Bewegungen wie den solidarischen Tong-Geheimgesellschaften in China und dem Piratentum. Sein mittlerweile in der anarchistischen Subkultur legendärer Ruf begründet sich auf seinen Broadsheets of Ontological Anarchism (1985) und Radio Sermonettes (1992). Bey regte konkrete Akte eines von ihm so bezeichneten poetischen Terrorismus an, um zum Beispiel Mainstream-Medien oder öffentlich bekannte Personen anzugreifen oder zu irritieren. Beys Lösungsansätze und anarchistischen Modelle liegen irgendwo zwischen urkommunistischen Gemeinschaftsformen "primitiver" Stämme und futuristischen Utopien einer postkapitalistischen Informationsgesellschaft (siehe sein Essay Primitive und Extropianer).

11 Gustav Landauer, „Durch Absonderung zur Gemeinschaft“, in: Ausgewählte Schriften, Bd. 7, hg. von Siegbert Wolf, Lich/Hessen, Verlag Edition AV, 2010, http://www.kontextverlag.de/landauer.html (19.10.2015).

12 TAZ: The Temporary Autonomous Zone, Ontological Anarchy, Poetic Terrorism (1991) (auf deutsch erschienen in der Edition ID-Archiv, Berlin, 1994). Siehe auch die online Ausgaben. 
Auch die Ideen einer ,revolutionären Romantik' rücken in den Vordergrund, insbesondere im Hinblick auf die Wechselwirkungen zwischen einer archaischen und einer utopischen Harmonie, zwischen der verlorenen und einer noch zu entstehenden freien Erfahrung, wovon u.a. zahlreiche Schriften des „sozialistischen Romantikers“(13) Gustav Landauer zeugen.

Immerhin haben um 1900 die Lebensreformer versucht, sich eine „andere Moderne“(14) auszudenken, vorwiegend indem sie beabsichtigten, Einzelmensch, Gemeinschaft und Umwelt auf neue Gleise zu stellen und mit Hilfe monistischer und holistischer, bzw. „naturgemäßer“(15) Auffassungen als Ganzes zu erfassen, wovon dann wieder die Schriften von Gustav Landauer zeugen, der sich immer wieder auf Meister Eckart beruft und immer wieder die Welt/Umwelt als Dialektik des Einzelnen und der Gesamtheit erfasst ${ }^{(16)}$. Selbstverständlich galt bei diesen Neuorientierungen ein besonderes Interesse den orientalischen Weisheiten, die dann auch den westlichen Erwartungen angepasst wurden. Um „das Unbehagen in der Kultur“ zu bekämpfen, gewann die Frage des Zusammenlebens an Interesse: Vorstellungen gemeinschaftlicher Übereinstimmung, eigentlich von sozialen „Wahlverwandtschaften“ bestimmt, wurden zum Zement des neuerdachten „sozialen Körpers“, in dem das „GesundeSchöne-Gute“ als Einheit erfasst wurde.

Der von den Lebensreformern ersehnte „große Neuansatz“ in der Zivilisation/Kultur implizierte eine neue Weltauffassung, deren Konzept eine Langzeitwirkung im Umfeld der Protestbewegungen hatte, man denke an die sog. Hippie- und New AgeBewegungen der Siebziger Jahre, oder an die zeitgenössische Globalisierungskritik, die seit 2000 laut wird und den utopischen Wunsch nach einer anderen Welt, und de facto nach einer anders verstandenen Globalisierung ${ }^{(17)}$ und selbstverständlich nach einer neuverstandenen „transnationalen Öffentlichkeit“ ausdrückt.

Die transnationale Öffentlichkeit bezeugt das Aufkommen einer völlig neuen Form von Mobilisierung, deren unaufhaltsame Ausbreitung die Staatsgrenzen sprengt, und zwar als „Gegenschlag“ zur rein wirtschaftlichen Globalisierung. Eng damit verbunden bleiben lebensreformerische Vorstellungen und Wünsche nach einer gesunden und „natürlichen“ körperlichen Lebensgestaltung, aber auch die Sehnsucht nach einem harmonischen solidarischen Zusammenleben und dem Aufkommen einer , anderen Welt', die zum großen Teil der Einführung einer "Gemeinwohlökonomie“ (Christian Felber $^{(18)}$ ) zu verdanken wäre. Religiöse und spirituelle/geistige Neuorientierungen sind aber auch an der Tagesordnung. Sie schlagen oft neue Wege ein, die vorwiegend

13 Justus H. Ulbricht, Romantik, Revolution und Reform, Göttingen, Wallstein Verlag, 1999.

14 Thomas Rohkrämer, Eine andere Moderne? Zivilisationskritik, Natur und Technik in Deutschland 1880-1933, Paderborn, Schöningh, 1999.

15 Eva BARLösıus, Naturgemäße Lebensführung: Zur Geschichte der Lebensreform um die Jahrhundertwende, Frankfurt am Main, Campus, 1997.

16 Vgl. Thorsten Hinz, Mystik und Anarchie. Meister Eckhart und seine Bedeutung im Denken Gustav Landauers, Berlin, Kramer, 2000; Walter Fähnders, „Sprachkritik und Wortkunst, Mystik und Aktion bei Gustav Landauer“, in: Jaap Grave, Peter Sprengel, Hans Vandevoorde (Hg.), Anarchismus und Utopie in der Literatur um 1900. Deutschland, Flandern und die Niederlande, Würzburg, Königshausen \& Neumann, 2005, S. 139-149. 
einen religiösen Synkretismus kennzeichnen. Sie orientieren sich wiederum stark an den dem Westen angepassten orientalischen Weisheiten, und sind eng verbunden mit dem Aufkommen eines Krisenbewusstseins, das sowohl die natürliche wie die soziale Umwelt betrifft. Dieses spirituelle/geistige Umdenken soll einen Lernprozess auslösen mit dem Ziel, anders zu denken, anders zu fühlen, anders zu wirken, wodurch Psyche, Körper und Umwelt/Weltall sich in Einklang entfalten können.

Da es jedoch immer fragwürdiger wird, ob eine „andere Welt“ überhaupt noch aufgebaut werden kann, hat sich der amerikanische Philosoph, eine der Hauptfiguren der Antiglobalisierungsbewegung, Hakim Bey, ein „ontologischer Anarchist“ und Sufi-Mystiker nach seiner Selbstdarstellung, die Möglichkeit der Entwicklung von PAZ und von TAZ, d.h. „Von permanenten und temporären autonomen Zonen“ ausgedacht. PAZ betreffen dann „villages“, „communes“, „communities“ und „Arcologies“, worunter eine ökologische Städtebauplanung verstanden wird, was dann auch wieder an die Gartenstadtbewegung um 1900 erinnert. TAZ sind befreit von jeder „kapitalistische[n] Hexerei“, wie es Isabelle Stengers formulieren würde ${ }^{(19)}$, und von jeder staatlichen Bevormundung, ganz in Resonanz mit der Art, wie sich die Lebensreformer in Arbeits- und Lebenskollektive zurückgezogen haben, um zu versuchen, ihre Ideale jenseits von Kapitalismus und Staat zu verwirklichen (man denke z.B. an die ziemlich bekannte „Edengenossenschaft“, die in Berlin Oranienburg 1893 gegründet wurde oder an die „Neue Gemeinschaft“(20), die kommunistisch-anarchistische Kommune am Berliner Schlachtensee, an deren Gründung 1900 Landauer teilnahm). In seinem Werk Dreißig Thesen zum Sozialismus erklärt Landauer in der These 16: „Die große Einheit ist zerrissen; eine Unzahl kleiner Geistesgemeinschaften ist da und will leben und hat keine notwendige Verbindung mit irgendeiner Gesamtkultur“(21).

Aber selbst wenn die PAZ eng mit den Idealen der lebensreformerischen Gemeinschaften in Verbindung stehen, besteht ein gewaltiger Unterschied zwischen ihnen und den TAZ, die unter dem Konzept „Immediatism“(22) zusammengefasst werden könnten. Für Hakim Bey entwirft die TAZ ein „ketzerisches Bewußtsein“, insofern als sie nur solange dauern sollen, wie die anarchistiche Ekstase anhält und dann zu verschwinden haben, da sie nur unkontinuierlich bestehen können. Kein Wunder also, dass gerade Seeräuber und Korsaren bzw. Piraten zu vorbildlichen Gestalten der TAZ werden, „da sie bewusst außerhalb des Gesetzes lebten und entschlossen waren durchzuhalten, sei es auch nur für eine kurze aber glückliche Zeit “(23), sprich eine lebendige

19 Isabelle Stengers, Philippe Pignarre, La sorcellerie capitaliste: Pratiques de désenvôutement, Paris, La Découverte, 2005.

20 Heinrich Hart, Julius Hart, Gustav Landauer, F. Holländer, Die Neue Gemeinschaft: ein Orden vom wahren Leben: Vorträge und Ansprachen, gehalten bei den Weihefesten, den Versammlungen und Liebesmahlen der Neuen Gemeinschaft, Leipzig, Eugen Diederichs Verlag, 1901. Siehe auch: Albert Weidner (Hg.), Die Neue Gemeinschaft. Mitteilungen fuer Mitglieder und Gleichgesinnte. Ein Reprintdruck in zwei Bänden, herausgegeben und kommentiert von R. F. Lang, Ortwig, Kulturhistorischer Verein Friedrichshagen e.V., Edition Friedrichshagen 12, 2011.

21 Gustav Landauer, Volk und Land: Dreißig sozialistische Thesen, 1907, www.anarchismus.at (12.09.2015).

22 Hakim BEY,Immediatism (1996), https://www.youtube.com/watch?v=9iWY_7Q3zXQ (12.09.2015).

23 Ebd. 
Zeit in einer lebendigen Welt. Das spontane Handeln wird dabei befürwortet: Ausdrücke wie Tatkraft, Aktionen, Aktivisten können für Stichwörter von Hakim Beys Vorstelllungen gelten, was aber dann auch wieder an Landauer erinnert, der schon im Jahre 1900 im bereits zitierten Werk Durch Absonderung zur Gemeinschaft schreibt, es gehe darum, „die Gemeinschaftswelt, die [er] meine, nicht wahrzunehmen, sondern sein und leben wollen“(24).

Nach Hakim Bey müssen unabhängige Enklaven oder anarchistische befreite Zonen geschaffen werden, die sich in der heutigen Technomedienwelt der Datenpiraterie widmen. Zonen oder Enklaven sind auch im Netz zu entwickeln, auch wenn nur für eine kurze Zeit. Unterstrichen wird dabei auch wieder der Zyklus von Erscheinen und Verschwinden, was nach seiner Auffassung nicht einem Sterben gleichzusetzen ist, sondern eher einem Untertauchen und Wiederauftauchen. Darin besteht die Freiheit des Einzelnen, das freie Wirken, wo der Einzelne zu Hause ist und woraus schließlich die Gemeinschaft besteht. Dabei geht es fast wieder um eine Landauersche Vorstellung: „Was der Mensch von Hause aus ist, was sein Innigstes und Verborgenstes, sein unantastbarstes Eigengut ist, das ist die große Gemeinschaft der Lebendigen in ihm [... $]^{\text {(25) }}$, schrieb Landauer in Durch Absonderung durch Gemeinschaft. Hakim Bey seinerseits betont mit besonderer Kraft das „Get a life, not a life style“-Prinzip, was an gewisse Denkmuster der Lebensreformer erinnern mag, denen zufolge das Erlangen der Harmonie in sich selbst (oder in einem eng begrenzten Kreis), die Harmonie des Ganzen fördern kann, sei es dank der „Ansteckungskraft des Guten“, sei es dank eines geheimnisvollen analog-magischen Effekts. S. Landauer:

„Die Gemeinschaft, nach der wir uns sehnen, die wir bedürfen, finden wir nur, wenn wir Zusammengehörige, wir neue Generation, uns von den alten Gemeinschaften absondern. Und wenn wir uns ganz gründlich absondern, wenn wir uns als Einzelne in uns selber tiefst hinein versenken, dann finden wir schließlich, im innersten Kern unseres verborgensten Wesens, die urälteste und allgemeinste Gemeinschaft: mit dem Menschengeschlecht und mit dem Weltall. Wer diese beglückende Gemeinschaft in sich selber entdeckt hat, der ist für alle Zeit bereichert und beseligt und endgültig abgerückt von den gemeinen Zufallsgemeinschaften der Mitwelt“(26).

Genau so geht es Hakim Bey nicht nur um die Entwicklung von Landeskommunen, die biologische Produkte anbauen. Selbst wenn Umweltbewusstsein und Ökologie („The living earth“) in seinem Werk zentral bleibt, geht es ihm keineswegs darum, die traditionelle Landidylle weiter zu pflegen:

„Wir haben kein Interesse ,zurück aufs Land zu gehen‘, wenn dies bedeutet das langweilige Leben eines sich plagenden Bauern zu führen - wir wollen auch keinen Tribalismus, wenn der mit Tabus, Fetischen und Unterernährung verbunden ist. Wir hadern nicht mit dem Konzept von Kultur - inklusive Technologie. Für uns beginnt das Problem mit Zivilisation. Was wir am paläologischen Leben mögen: die elegante Faulheit der Jäger/SammlerGesellschaft, der 2-Stunden Arbeitstag, das Besessensein von Kunst, Tanz, Poesie und

24 G.. LANDAuer, „Durch Absonderung zur Gemeinschaft“ (Anm. 11). 
Liebe, die Demokratisierung des Schamanismus, die Kultivierung der Wahrnehmung, kurz Kultur. Was wir an Zivilisation nicht ausstehen können: die agrarische Revolution, das Entstehen der Kaste, die Stadt und ihr Kult hieratischer Kontrolle, Sklaverei, Dogma, Imperialismus, die Unterdrückung von Sexualität unter der Ägide von Autorität. [...] Das Imperium hat nie aufgehört“(27).

In diesem Zitat können lebensreformerische Themen aufgezählt werden, selbst wenn sie etwas modernisiert bzw. postmodernisiert worden sind. Hakim Bey nähert sich dabei der Empirerepräsentation, wie sie Michael Hardt und Antonio Negri in ihrem bereits erwähnten Werk ausgearbeitet haben. Die Zeit der unfassbaren und unbegrenzten Staats- oder Empiregewalt, eine Zeit in der die Omnipräsenz und Allmacht des Staates/Empire behauptet wird, muss zugleich Risse und Leerstellen aufzeigen. Die TAZ ist daher eine perfekte, der zeitlichen Entwicklung angepasste Taktik: das Untemporale, Kurzfristige ist bei Hakim Bey ausschlaggebend. Sobald die TAZ benannt, repräsentiert oder mediatisiert wird, muss sie untertauchen und sozusagen die Öffentlichkeit überwinden.

Weder für die Lebensreformer noch für die Globalisierungskritiker à la Hakim Bey geht es vorrangig um eine radikale Veränderung der Welt, sondern vielmehr um ein dezidiertes Umdenken: „Der Realismus verlangt nicht nur, dass wir das Warten auf die Revolution aufgeben, sondern aufhören sie zu wollen“(28), schreibt Hakim Bey, ganz in Resonanz mit Landauers Überzeugungen: „Wir warten nicht auf die Revolution, damit dann der Sozialismus beginne; sondern wir fangen an, den Sozialismus zur Wirklichkeit zu machen, damit dadurch der große Umschwung komme ${ }^{(29)}$. Keine sofortige Revolution wird erhofft, sondern Aufstand wird befürwortet, und zwar so oft wie möglich. „Eine nomadische Kriegsmachinerie“(30) soll in Gang gesetzt werden, die als „eine okkulte Kunst innerhalb der Kampfsportarten“ verstanden wird ${ }^{(31)}$. „Der erste Schritt ist Satori ähnlich“(32) schreibt Hakim Bey. Landauer verankerte seinerseits seine Überlegungen im Werk von Meister Eckart, berief sich aber auch öfters auf Buddha.

Immerhin scheint Hakim Bey, wie Gustav Landauer zu seiner Zeit, eine bedeutende Rolle zu spielen, vorwiegend im Rahmen der Neubelebung eines möglichen Zusammenschmelzens von Reform/Revolution und Spiritualität, von Anarchie und abendländischer sowie altorientalischer Weisheit. Landauer und Hakim Bey können also als Vordenker und Propheten eines transnationalen reformerischen, also auch de facto utopischen Denkens ${ }^{(33)}$ („Zurück zur Utopie“) gelten, das beabsichtigt, menschlichen Kontakt im Rahmen eines gemeinschaftlichen Lebens wiederherzustellen im Sinne

27 H. BEy, TAZ (Anm. 12), S. 52.

28 Ebd.

29 Gustav Landauer, Auch die Vergangenheit ist Zukunft. Essays zum Anarchismus, hg. von Siegbert Wolf, Darmstadt, Luchterhand, 1989, S. 198ff., zitiert in: Lexikon deutsch-jüdischer Autoren, Bd. 15, München, 2007, S. 53.

30 H. Bey, TAZ (Anm. 12), S. 144.

31 Ebd.

32 Ebd. Satori ist die Erkenntnis vom universellen Wesen des Daseins, das auch als Urgrund oder Buddha-Natur bezeichnet wird. Es ist das Hauptmotiv des Zen-Buddhismus und kann nur durch persönliche Erfahrung verstanden werden.

33 Hakim BEY, Permanent TAZ, http//hermetic.com/bey/paz.html. 
der von Ivan Illich geprägten „conviviality“, „to restore human contact “(34), wobei ihre spirituelle anarchistische „Resonanz" wieder deutlich zum Vorschein kommt. Es bleibt jedoch die Frage offen, ob die alternativen Bewegungen schließlich doch nicht den Keim der Anpassung an das verhasste System in sich bergen. Geht es eigentlich nicht um „Rebellionen der Anpassung“, ob transnational oder nicht, die dem Einzelnen, sowohl um 1900 wie um 2000, die Möglichkeit bieten, in „alternativen Nischen“ ihr Leben „anders“ zu gestalten, wobei sie sich keineswegs dem Strom des verhassten Fortschritts und dem Empire widersetzen, sondern ganz im Gegenteil sie sogar fördern. Transnationale Öffentlichkeit, als öffentliche Meinungs- und Willensbildung verstanden, die meist auch für Kriterien einer funktionierenden Demokratie gelten, kann also auch den Keim einer undemokratischen bzw. ,totalitären' Anpassung an das in der Öffentlichkeit verworfene System enthalten, und verhilft letzterem weiter zu funktionieren.

\section{Zusammenfassung}

Im Kontext der transnationalen Öffentlichkeit, hier diachronisch erfasst, scheint die Antiglobalisierungsbewegung, wie sie sich seit 2000 entwickelt hat, das Ideengut der um 1900 entstandenen Lebensreformbewegungen wieder aufzunehmen. Diese Bewegungen versuchten eine harmonische und naturgemäße Entwicklung des Einzelnen zu ermöglichen, im Rahmen eines gesellschaftlichen Ganzen, wobei die Ideen einer, revolutionären Romantik'befürwortet wurden. Der Beitrag wird die Schriften des „sozialistischen Romantikers" Gustav Landauer (1870-1919) mit denen von Hakim Bey (eigentlich Peter Lamborn Wilson, 1945 in New York geboren), einer der Hauptfiguren der Antiglobalisierungsbewegung, sozusagen, in Resonanz' analysieren, wobei auf die Rolle einer Gegenöffentlichkeit, die sich als Resistenz gegenüber der heutigen transnationalen Technomedienwelt versteht, fokussiert wird.

\section{Résumé}

Nous proposons d'aborder la notion d'espace public transnational dans sa dimension diachronique en interrogeant les mouvements altermondialistes, tels qu'ils se sont développés autour de 2000 qui semblent ancrés dans un fonds de pensée issu des mouvements de la réforme de la vie (Lebensreform). Ces mouvements cherchèrent à promouvoir un développement harmonieux et "naturel» de l'individu saisi dans sa relation au groupe social et revivifièrent pour cela un "romantisme révolutionnaire». Cette contribution se propose d'aborder en résonance les textes du "socialiste romantique» Gustav Landauer (1870-1919) et ceux de Hakim Bey (en réalité Peter Lamborn Wilson, né en 1945 à New York), figure de proue de l'altermondialisme, et de mettre l'accent sur le rôle d'un "contre espace public» compris comme le lieu de résistance à un espace public technomédiatique, de facto transnational. 


\begin{abstract}
In the context of a transnational publicity, which is here measured diachronically, the anti-globalization movement, as it has been developing since the year 2000, seems to readopt the ideas of the "Lebensreform" - movements which were established around 1900. These movements tried to enable a harmonic and natural development of the individual within the frame of the society, supporting the ideas of a, revolutionary romanticism. This article will analyze, in response (oder in resonance) the writings of the "socialistic romanticist" Gustav Landauer (1870-1919) and compare them to those of Hakim Bey (originally Peter Lamborn Wilson, born in 1945 in New York), one of the central characters of the anti-globalization movement, focussing on the role of an anti-publicity which is to be understood as a resistance against todays transnational world of techno-media.
\end{abstract}

